\title{
Critical points in two-channel quantum systems
}

\author{
Hichem Eleuch ${ }^{1,2}$ and Ingrid Rotter ${ }^{3, a}$ \\ ${ }^{1}$ Institute for Quantum Science and Engineering, Texas A\&M University, College Station, Texas 77843, USA \\ 2 Department of Applied Sciences and Mathematics, College of Arts and Sciences, Abu Dhabi University, \\ Abu Dhabi, UAE \\ ${ }^{3}$ Max Planck Institute for the Physics of Complex Systems, 01187 Dresden, Germany
}

Received 24 January 2018 / Received in final form 1 June 2018

Published online 7 August 2018

(C) The Author(s) 2018. This article is published with open access at Springerlink.com

\begin{abstract}
Calculations for open quantum systems are performed usually by taking into account their embedding into one common environment, which is mostly the common continuum of scattering wavefunctions. Realistic quantum systems are coupled however mostly to more than one continuum. For example, the conductance of an open cavity needs at least two environments, namely the input and the output channel. In the present paper, we study generic features of the transfer of particles through an open quantum system coupled to two channels. We compare the results with those characteristic of a one-channel system. Of special interest is the parameter range which is influenced by singular points. Here, the states of the system are mixed via the environment. In the one-channel case, the resonance structure of the cross section is independent of the existence of singular points. In the two-channel case, however, new effects appear such as coherence. An example is the enhanced conductance of an open cavity in a certain finite parameter range. It is anti-correlated with the averaged phase rigidity of the eigenfunctions of the non-Hermitian Hamilton operator.
\end{abstract}

\section{Introduction}

Quantum systems are localized in a finite well-defined space area. This area may be determined by certain boundary conditions (e.g. in quantum dots or quantum billiards) or by self-organization (e.g. in atomic nuclei). The shape of the nucleus is, indeed, an essential part of nuclear spectroscopic investigations and is characteristic of every nucleus.

The spectroscopic properties of quantum systems are studied usually by means of a Hermitian Hamilton operator $H$, the eigenstates of which are discrete. Due to the embedding of the system into a continuum of scattering wavefunctions, the lifetimes of its states become usually finite. This agrees with the experimentally well-known finite lifetime of the states of a quantum system which is described in the standard Hermitian quantum theory by means of the tunneling mechanism. An exception are the well-known bound states in the continuum the lifetime of which is infinitely long. These states occur under special conditions and are described well in different papers for different systems, e.g. [1-4].

Moreover, the embedding of a quantum system into a continuum of scattering wavefunctions causes another effect which is not contained in standard Hermitian quantum theory: since every state of the system is coupled to

\footnotetext{
${ }^{\mathrm{a}}$ e-mail: rotter@pks.mpg.de
}

the continuum, all states of the system may mix via the environment. This so-called external mixing (EM) of the states is a second-order process and occurs additionally to the well-known direct (internal) mixing of the states which is contained in every Hermitian Hamiltonian $H$. In the standard Hermitian calculations, EM is effectively taken into account as an additional contribution to the internal mixing. Its characteristic features are lost in this case.

Experimentally, an example of EM has been provided a few years ago in a mesoscopic system. It has been shown in [5] that two distinct quantum states are coupled through a common continuum. In a further experiment, the authors were able to show that EM survives even under conditions of strongly non-equilibrium transport in the system $[6]$.

A possible EM of quantum states is very seldom considered in theory. It can easily be taken into account when the properties of the system are described by a non-Hermitian Hamiltonian $\mathcal{H}$. In such a case, EM is involved explicitly in the eigenfunctions of $\mathcal{H}$, see [7-11].

In [11] the, at first glance, unexpected result has been found that EM needs not to be considered when the system is embedded in a common environment of scattering wavefunctions. This result corresponds to the experience obtained from many numerical studies on realistic systems in which EM is not at all considered. Also the influence of the singular exceptional points (EP) is not involved in these calculations. 
Nevertheless, these results are not in contradiction with the statement of non-Hermitian quantum physics that EPs and EM may influence, to a great extent, the spectroscopic properties of open quantum systems. The point is that EPs cause nonlinear processes in their neighborhood [7-9] which are able to compensate the contributions that arise from the EM of the states via the common environment [11]. Correspondingly, the cross section shows the same resonance structure in the one-channel case when calculated with EPs and EM or without them.

The situation is however different when the system is embedded not only in one common environment, but in several environments which exist independently of one another. An example of such a situation is the decay of a nuclear state into different states of the residual nucleus by emission of a nucleon. In such a case, we have the different so-called partial widths that characterize the decay of the state $i$ of the system in one of the open channels $c$. The different channels $c$ are defined by the decay of the state $i$ into different states of the residual nucleus by emission of a nucleon. A channel is open when the energy difference between the state $i$ and the state of the residual nucleus is positive, i.e. when the emission of a particle is allowed.

Another - and more important - example is the transmission through, e.g., a quantum dot. In this case, at least two different environments exist, namely that of the entrance channel and that of the exit channel. Several numerical studies performed with a non-Hermitian Hamilton operator $\mathcal{H}$ on the basis of the tight-binding model have shown interesting non-trivial results $[12,13]$ : the transmission probability is anti-correlated with the phase rigidity of the eigenfunctions of the non-Hermitian Hamilton operator (when averaged over energy in a certain energy window). That means it depends on internal properties of the eigenfunctions of $\mathcal{H}$.

An enhancement of transmission through quantum dots or quantum billiards is found also in other studies, mostly by varying the coupling strength of the system to the environment. In $[14,15]$, complex scaling is used while in $[16,17]$ the formation of a so-called superradiant state is considered. In [12-15], the enhancement of the transmission is related to the properties of the eigenfunctions of the non-Hermitian Hamilton operator $\mathcal{H}$.

We mention here that the effects, which an environment has on the transmission of particles through a quantum dot, have been considered also in, e.g., [18]. In this paper, the competing effects of Markovian and non-Markovian mechanisms have been investigated.

It is the aim of the present paper to find typical spectroscopic features of an open quantum system that is coupled to two particle-decay channels, which are independent of one another. In Section 2 we discuss the effective non-Hermitian Hamiltonian $H^{\mathrm{eff}}$ which is used often in describing realistic systems. In Section 3, we sketch the formalism of the genuine non-Hermitian Hamiltonian; and in Section 4 we show some typical numerical results. A discussion of the obtained results can be found in Section 5. Their relation to the results of other studies with non-Hermitian Hamiltonians is also discussed. Concluding remarks can be found in Section 6 .

\section{Effective non-Hermitian Hamilton operator $\boldsymbol{H}^{\text {eff }}$}

Transfer and other processes with excitation of individual resonance states are described successfully in non-Hermitian quantum physics by using the effective Hamiltonian [7]

$$
H^{\mathrm{eff}}=H_{0}+\sum_{c} V_{0 c} \frac{1}{E^{+}-H_{c}} V_{c 0} .
$$

Here, $H_{0}$ is the Hamiltonian describing the corresponding closed system with discrete states, $\left(E^{+}-H_{c}\right)^{-1}$ is the Green function in the continuum with the Hamiltonian $H_{c}$ describing the environment of decay channels, and $V_{0 c}, V_{c 0}$ stand for the coupling of the closed system to the different channels $c$ of the environment. The nonHermiticity of $H^{\text {eff }}$ arises from the second term of $H^{\text {eff }}$, i.e. from the perturbation of the system occurring under the influence of its coupling to the environment. It is complex: the real part arises from the principal value integral and the imaginary one from the residuum (for details see [7]).

Sometimes, $H^{\text {eff }}=H_{0}+\alpha W$ is assumed where $W$ is imaginary, and the properties of the system are studied as a function of $\alpha$ (for examples see the review [7]). In [19], the non-Hermitian operator is assumed to be $\hat{\mathcal{H}}=\hat{H}-i \hat{\Gamma}$. This operator allows us to study the general meaning of the imaginary part of the non-Hermitian Hamiltonian, i.e. dissipation. The information on the considered physical system (embedded into a well-defined environment), which is involved in $H^{\text {eff }}$, is however lost. In any case, the non-Hermiticity of $H^{\text {eff }}$ arises from the second term which is added to $H_{0}$ as a perturbation.

The eigenfunctions of a non-Hermitian Hamilton operator are biorthogonal

$$
\mathcal{H}\left|\Phi_{i}\right\rangle=\mathcal{E}_{i}\left|\Phi_{i}\right\rangle \quad\left\langle\Psi_{i}\right| \mathcal{H}=\mathcal{E}_{i}\left\langle\Psi_{i}\right| .
$$

In the case of the symmetric Hamiltonian $H^{\text {eff }}$, it is

$$
\Psi_{i}=\Phi_{i}^{*},
$$

and the eigenfunctions should be normalized according to

$$
\left\langle\Phi_{i}^{*} \mid \Phi_{j}\right\rangle=\delta_{i j},
$$

in order to smoothly describe the transition from a closed system with discrete states to a weakly open one with narrow resonance states. As a consequence of (4), the values of the standard expressions are changed,

$$
\begin{aligned}
\left\langle\Phi_{i} \mid \Phi_{i}\right\rangle & =\operatorname{Re}\left(\left\langle\Phi_{i} \mid \Phi_{i}\right\rangle\right) ; \quad A_{i} \equiv\left\langle\Phi_{i} \mid \Phi_{i}\right\rangle \geq 1, \\
\left\langle\Phi_{i} \mid \Phi_{j \neq i}\right\rangle & =i \operatorname{Im}\left(\left\langle\Phi_{i} \mid \Phi_{j \neq i}\right\rangle\right)=-\left\langle\Phi_{j \neq i} \mid \Phi_{i}\right\rangle, \\
\left|B_{i}^{j}\right| & \equiv \mid\left\langle\Phi_{i}\left|\Phi_{j \neq i}\right| \geq 0\right.
\end{aligned}
$$

The advantage to describe the properties of the open system by means of $H^{\text {eff }}$ consists, above all, in the possibility to use the results obtained for the corresponding 
closed system. In both cases, the properties are determined by nothing but the eigenstates of the many-body system. Usually, experimental results on inelastic scattering and transfer of particles through a small system are described well by $H^{\text {eff }}$ (examples can be found in [7]).

In (1), a singular point may appear, the so-called exceptional point (EP), at which two eigenvalues of $H^{\text {eff }}$ coalesce [20]. At these points, the two corresponding eigenfunctions are not orthogonal. Instead

$$
\Phi_{1}^{\mathrm{cr}} \rightarrow \pm i \Phi_{2}^{\mathrm{cr}} ; \quad \Phi_{2}^{\mathrm{cr}} \rightarrow \mp i \Phi_{1}^{\mathrm{cr}},
$$

according to analytical and numerical results [21-25]. An $\mathrm{EP}$ is, according to its definition, related to the common environment in which the system is embedded. In other words, it is well defined under the condition that the system is embedded in only one continuum.

Far from EPs, the coupling of the localized system to the environment influences the spectroscopic properties of the system only marginally $[7,11]$. The influence is however nonvanishing also in this case, see e.g. the theoretical results [26] for very small coupling strength between system and environment, which are proven experimentally [27]. These experimental results cannot be described by $H^{\text {eff }}$.

Another deficit of $H^{\text {eff }}$ is that this formalism cannot be used for the description of systems with transfer of excitons. An example is the photosynthesis in which not any eigenstates are excited, see [28].

We will consider therefore in the next Section 3 a genuine non-Hermitian Hamilton operator $\mathcal{H}$ which is much less convenient for numerical calculations than $H^{\text {eff }}$. It gives us however a deeper insight into the reordering processes occurring in open quantum systems under the influence of the singular EPs.

\section{Genuine non-Hermitian Hamiltonian $\mathcal{H}$}

\subsection{Eigenvalues and eigenfunctions of $\mathcal{H}^{(2,1)}$}

To begin with, we sketch the features typical for an open quantum system embedded in one common continuum. Details can be found in [7] and, above all, in [11]. They can be discussed by means of the $2 \times 2$ genuine non-Hermitian matrix

$$
\mathcal{H}^{(2,1)}=\left(\begin{array}{cc}
\varepsilon_{1}^{(1)} \equiv e_{1}^{(1)}+\frac{i}{2} \gamma_{1}^{(1)} & \omega^{(1)} \\
\omega^{(1)} & \varepsilon_{2}^{(1)} \equiv e_{2}^{(1)}+\frac{i}{2} \gamma_{2}^{(1)}
\end{array}\right) .
$$

Here, the $e_{i}^{(1)}$ are the energies of the localized states $i$ and the $\gamma_{i}^{(1)}$ are their widths. ${ }^{1}$ The $\omega^{(1)}$ stand for the coupling matrix elements of the two states via the common environment (1). They are complex where $\operatorname{Re}\left(\omega^{(1)}\right)$ arises from the

\footnotetext{
${ }^{1}$ In contrast to the definition that is used in, for example, nuclear physics, we define the complex energies before and after diagonalization of $\mathcal{H}$ by $\varepsilon_{k}=e_{k}+\frac{i}{2} \gamma_{k}$ and $\mathcal{E}_{k}=E_{k}+\frac{i}{2} \Gamma_{k}$, respectively, with $\gamma_{k} \leq 0$ and $\Gamma_{k} \leq 0$ for decaying states. This definition will be useful when discussing systems with gain (positive widths) and loss (negative widths).
}

principal value integral and $\operatorname{Im}\left(\omega^{(1)}\right)$ from the residuum [7]. The complex eigenvalues $\mathcal{E}_{i}^{(1)} \equiv E_{i}^{(1)}+\frac{1}{2} \Gamma_{i}^{(1)}$ of $\mathcal{H}^{(2,1)}$ give the energies $E_{i}^{(1)}$ and widths $\Gamma_{i}^{(1)}$ of the states of the localized part of the system. We call the operator $\mathcal{H}^{(2,1)}$ genuine since it is not related directly to any special quantum system (in contrast to (1)). It contains nothing but two states characterized by their complex energies $\varepsilon_{i}$ and their coupling matrix elements $\omega$ via the environment.

The eigenfunctions of $\mathcal{H}$ are biorthogonal, see (2)-(6). It is meaningful to define the phase rigidity which is a quantitative measure for the biorthogonality of the eigenfunctions,

$$
r_{k} \equiv \frac{\left\langle\Phi_{k}^{*} \mid \Phi_{k}\right\rangle}{\left\langle\Phi_{k} \mid \Phi_{k}\right\rangle}=A_{k}^{-1} .
$$

It is smaller than 1 . Far from an EP, $r_{k} \approx 1$ while it approaches the value $r_{k}=0$ when an EP is approached.

Additionally to the Hamiltonian (8), we will consider the non-Hermitian matrix

$$
\mathcal{H}_{0}^{(2,1)}=\left(\begin{array}{cc}
\varepsilon_{1}^{(1)} \equiv e_{1}^{(1)}+\frac{i}{2} \gamma_{1}^{(1)} & 0 \\
0 & \varepsilon_{2}^{(1)} \equiv e_{2}^{(1)}+\frac{i}{2} \gamma_{2}^{(1)}
\end{array}\right),
$$

which describes the system without any mixing of its states via the environment. In other words, $\omega=0$ corresponds to vanishing EM of the eigenstates. The eigenfunctions $\Phi_{i}$ of $\mathcal{H}^{(2,1)}$ can be represented in the set of eigenfunctions $\left\{\Phi_{i}^{0}\right\}$ of $\mathcal{H}_{0}^{(2,1)}$,

$$
\Phi_{i}=\sum b_{i j} \Phi_{j}^{0} ; \quad b_{i j}=\left\langle\Phi_{j}^{0 *} \mid \Phi_{i}\right\rangle,
$$

under the condition that the $b_{i j}$ are normalized by $\sum_{j}\left(b_{i j}\right)^{2}=1$. The coefficients $\left|b_{i j}\right|^{2}$ differ from the $\left(b_{i j}\right)^{2}$. They contain the information on the strength of EM.

The main features characteristic of open quantum systems are described well by the eigenvalues and eigenfunctions of (8). Typical values related to the eigenfunctions are the phase rigidity (9) and the contribution of EM (11) to their purity. All these values contain the influence of the environment. They are proven experimentally, for details see $[7,11]$ and above all the review [29].

\subsection{Schrödinger equation with $\mathcal{H}^{(2,1)}$}

The Schrödinger equation $\left(\mathcal{H}^{(2,1)}-\mathcal{E}_{i}^{(1)}\left|\Phi_{i}^{(1)}\right\rangle=0\right.$ may be rewritten into a Schrödinger equation with source term $[7,11]$,

$$
\left(\mathcal{H}_{0}^{(2,1)}-\mathcal{E}_{i}^{(1)}\right)\left|\Phi_{i}^{(1)}\right\rangle=-\left(\begin{array}{cc}
0 & \omega \\
\omega & 0
\end{array}\right)\left|\Phi_{i}^{(1)}\right\rangle .
$$

In this representation, the coupling $\omega$ of the states $i$ and $j \neq i$ of the localized system via the common environment of scattering wavefunctions (EM) is contained in the source term of the Schrödinger equation, for details see [7].

Far from EPs, the coupling of the localized system to the environment influences the spectroscopic properties of 
the system only marginally $[7,11]$. The influence is however nonvanishing also in this case, see e.g. the experimental results [27] for the case that the coupling between system and environment is very small.

In the neighborhood of EPs, the coupling between system and environment causes - according to mathematical studies - nonlinear effects in the Schrödinger equation (12), see [7,11]. Among others, these effects lead to a conservation of the resonance structure of the cross section in the one-channel case which is therefore unaffected by EM and by the existence of EPs. Thus, the one-channel case cannot be used in order to test the results of the non-Hermitian formalism.

\subsection{Eigenvalues and eigenfunctions of $\mathcal{H}^{(2,2)}$}

Let us now consider the $4 \times 4$ non-Hermitian matrix

$$
\mathcal{H}^{(2,2)}=\left(\begin{array}{cccc}
\varepsilon_{1}^{(1)} & \omega^{(1)} & 0 & 0 \\
\omega^{(1)} & \varepsilon_{2}^{(1)} & 0 & 0 \\
0 & 0 & \varepsilon_{1}^{(2)} & \omega^{(2)} \\
0 & 0 & \omega^{(2)} & \varepsilon_{2}^{(2)}
\end{array}\right) .
$$

Here, $\varepsilon_{i}^{(1)} \equiv e_{i}^{(1)}+\frac{i}{2} \gamma_{i}^{(1)}$ and $\varepsilon_{i}^{(2)} \equiv e_{i}^{(2)}+\frac{i}{2} \gamma_{i}^{(2)}$ are the complex energies of the localized states $i$ relative to channel 1 and 2, respectively (see Footnote 1 ). Usually $\varepsilon_{i}^{(1)} \neq$ $\varepsilon_{i}^{(2)}$. The $\omega^{(1)}$ and $\omega^{(2)}$ stand for the coupling matrix elements of the two states via the environment 1 and 2, respectively. The Hamiltonian (13) includes the fact that the complex energy $\varepsilon_{i}^{(c)}$ of the localized state $i$ is different relative to the two different channels $c$.

It might be astonishing that the Hamiltonian (13) contains four states instead of the original two states. The point is the following. Two states may mix, independently of one another, via each of the two environments with the result that the energies $\varepsilon_{i}^{(c)}$ depend not only on the state number $i$ but also on the channel $c$. That means, every state is formally doubled, since it is embedded into two different environments (channels). The two environments are different from and orthogonal to one another. Further, the two states with equal $i$ and different $c$ arise from the same state $i$ of the localized part of the system. The zeros in the matrix (13) express the corresponding fact that the two states $i$ relative to the two channels 1 and 2 cannot interact with one another.

The eigenvalues $\mathcal{E}_{i}^{(c)} \equiv E_{i}^{(c)}+\frac{i}{2} \Gamma_{i}^{(c)}$ and eigenfunctions $\Phi_{i}^{(c)}$ of (13) are characterized also by two numbers: the number $i$ of the state $(i=1,2)$ of the localized part of the system and the number $c$ of the channel $(c=1,2)$, called environment, in which the system is embedded. Usually, $E_{i}^{(1)} \neq E_{i}^{(2)}$ and $\Gamma_{i}^{(1)} \neq \Gamma_{i}^{(2)}$. Also the wave functions $\Phi_{i}^{(1)}$ and $\Phi_{i}^{(2)}$ differ from one another due to the EM of the eigenstates via the environment 1 and 2 , respectively. That means, the system described by (13) has formally four states (from a mathematical point of view). The original two states related to the values $i=1$ and $i=2$ are doubled due to the fact that each state $i$ is coupled to the two channels $c=1$ and $c=2$.
We mention further that the Hamiltonian (13) is formally the same as the Hamiltonian (24) or rather (1) in [28]. There is however a fundamental difference: in (1) in [28], the transition of excitons (expressed by fluctuations caused by EPs) is considered while (13) describes the transition of particles. Furthermore, in (1) in [28] the whole system is fully embedded into both environments (1) and (2) which both are of completely different nature and exist independently of one another. In contrast to this, the Hamiltonian (13) describes a system, the states of which are embedded partially in each of the two different environments. These two different environments are also independent of one another. They are, however, nothing but parts of the total environment.

Without singularity in the considered parameter range in relation to both channels, we have $E_{i}^{(1)} \approx E_{i}^{(2)}, \Gamma_{i}^{(1)} \approx$ $\Gamma_{i}^{(2)}$ and $\Phi_{i}^{(1)} \approx \Phi_{i}^{(2)}$. This case is realized in the decay of nuclear states since their decay probability can be determined only when the decaying state is well isolated from other states. Otherwise, new problems arise from the overlapping with other states $[30,31]$. The above case is realized also in the transmission when the individual transmission peaks are well isolated from one another.

Under the influence of a singularity relative to $c=1$ and/or relative to $c=2$, the eigenvalues and eigenfunctions will be, however, different from one another, $E_{i}^{(1)} \neq$ $E_{i}^{(2)}, \Gamma_{i}^{(1)} \neq \Gamma_{i}^{(2)}$ and $\Phi_{i}^{(1)} \neq \Phi_{i}^{(2)}$ in the corresponding parameter range. Such a situation may occur in the transmission through a quantum dot or quantum billiard $[12,13]$.

In analogy to (10), we will consider also the nonHermitian Hamiltonian

$$
\mathcal{H}_{0}^{(2,2)}=\left(\begin{array}{cccc}
\varepsilon_{1}^{(1)} & 0 & 0 & 0 \\
0 & \varepsilon_{2}^{(1)} & 0 & 0 \\
0 & 0 & \varepsilon_{1}^{(2)} & 0 \\
0 & 0 & 0 & \varepsilon_{2}^{(2)}
\end{array}\right),
$$

which describes the system without any mixing of its states via any environment. In other words, $\omega^{(1)}=$ $\omega^{(2)}=0$ corresponds to vanishing EM of the eigenstates via an environment. The mixing of the eigenstates of (13) can be represented in a set of eigenfunctions of (14) in complete analogy to the relation (11) for two states coupled to one common environment.

\subsection{Schrödinger equation with $\mathcal{H}^{(2,2)}$}

Using (14), we can write down the Schrödinger equation with source term for the two-channel case in analogy to (12) for the one-channel case. The corresponding equation reads

$$
\left(\mathcal{H}_{0}^{(2,2)}-\mathcal{E}_{i}^{(c)}\right)\left|\Phi_{i}^{(c)}\right\rangle=-\left(\begin{array}{cccc}
0 & \omega^{(1)} & 0 & 0 \\
\omega^{(1)} & 0 & 0 & 0 \\
0 & 0 & 0 & \omega^{(2)} \\
0 & 0 & \omega^{(2)} & 0
\end{array}\right)\left|\Phi_{i}^{(c)}\right\rangle .
$$


The source term depends on the coupling of the system to both channels, i.e. on $\omega^{(1)}$ and on $\omega^{(2)}$. It does not depend on the energies $\varepsilon_{i}^{(c)}$.

We repeat here that, according to their definition [20], EPs occur only in the one-channel case, i.e. only in the two submatrices related to channel 1 and channel 2, respectively. They are not defined in the $4 \times 4$ matrix (13). However, the EPs of the two submatrices in (13) may influence the dynamics of the open two-channel system.

\subsection{Non-Hermitian Hamiltonian and resonance structure of the $S$ matrix}

An expression for the $S$ matrix is derived and discussed in detail in [32] by rewriting the Breit-Wigner expression for one or more isolated resonances. According to this derivation, the resonance structure of the $S$ matrix containing two resonance states, can be obtained from the expression

$$
S=\frac{\left(E-E_{1}-\frac{i}{2} \Gamma_{1}\right)\left(E-E_{2}-\frac{i}{2} \Gamma_{2}\right)}{\left(E-E_{1}+\frac{i}{2} \Gamma_{1}\right)\left(E-E_{2}+\frac{i}{2} \Gamma_{2}\right)},
$$

for the case that two resonance states are coupled to a common continuum of scattering wavefunctions, see also $[7,11]$. The expression (16) is unitary. According to (16), the resonance structure of the cross section is determined exclusively by the spectroscopic values of the localized part of the system, i.e. by the eigenvalues $\mathcal{E}_{i}=E_{i}+i / 2 \Gamma_{i}$ of the non-Hermitian Hamiltonian $\mathcal{H}$. The expression (16) allows us therefore to obtain reliable results for the two channels when the phase rigidity of the eigenfunctions of $\mathcal{H}$ is reduced $\left(r_{k}<1\right)$ and when the eigenfunctions of $\mathcal{H}$ contain EM, i.e. when they are mixed in the set of eigenfunctions $\left\{\Phi_{i}^{0}\right\}$ of $\mathcal{H}_{0}$ according to (11).

As shown in $[7,11]$, the $S$ matrix contains, generally, nonlinear effects which are caused by the EM of the resonance states via the environment. The one-channel case does, however, not allow us to prove the existence of these nonlinear effects and of EM, since the resonance structure of the cross section calculated with and without EM is the same in this case [11]. This result agrees, on the one hand, with the experience received from many different numerical studies in realistic cases which are performed without taking into account EM. On the other hand, it is not in contradiction with the conclusions received from the study of non-Hermitian physics of open quantum systems for the following reason.

According to the results obtained in [11] for the onechannel case, the evolution of the system near to an EP is driven exclusively by the nonlinear source term of the Schrödinger equation (12) which describes the coupling of the localized part of the system to the common environment and is characteristic of the open quantum system embedded in one environment. The calculations in [11] are performed without varying $\omega$, i.e. $\omega$ can not be responsible for the width bifurcation occurring in these calculations under the influence of an EP. Obviously, the nonlinear source term is the driving force. It is able, in the onechannel case, to largely conserve the resonance structure of the cross section.
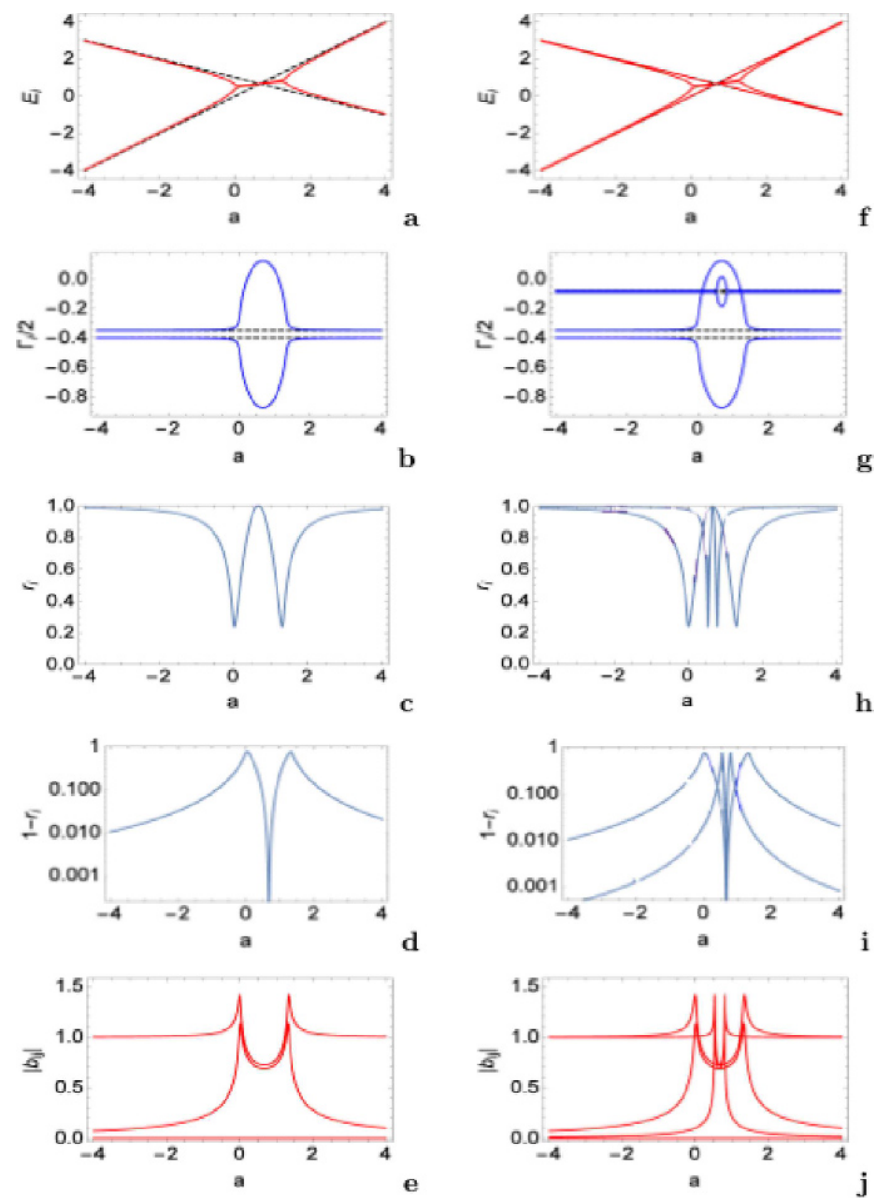

Fig. 1. Eigenvalues $\mathcal{E}_{i}^{(1,2)} \equiv E_{i}^{(1,2)}+\frac{i}{2} \Gamma_{i}^{(1,2)}$ and eigenfunctions $\Phi_{i}^{(1,2)}$ of the Hamiltonian $\mathcal{H}^{(2,2)}$ as a function of $a$. $\omega^{(1)}=\omega^{(2)}=0.5 i$ (left), $\omega^{(1)}=0.5 i ; \omega^{(2)}=0.1 i$ (right). Parameters: $e_{1}=1-a / 2 ; e_{2}=a$; left: $\gamma_{1}^{(1)} / 2=-0.4 ; \gamma_{1}^{(2)}=$ $-0.35 ; \gamma_{2}^{(1)} / 2=-0.35 ; \gamma_{2}^{(2)} / 2=-0.4($ dashed lines in $(\mathrm{a}, \mathrm{b}))$; right: $\gamma_{1}^{(1)} / 2=-0.4 ; \gamma_{1}^{(2)}=-0.08 ; \gamma_{2}^{(1)} / 2=-0.35 ; \gamma_{2}^{(2)} / 2=$ -0.09 (dashed lines in (f, g)). At the critical parameter value $a=a^{\text {cr }}=0.6494$, the phase rigidity $r_{i}$ approaches the value 1 .

The conservation of the resonance structure of the cross section which is possible in the one-channel case, is expected to be impossible, generally, in the two-channel (or more-channel) case according to (15) since, generally, $\omega^{(1)} \neq \omega^{(2)} \neq 0$. This gives the chance to test nonHermitian quantum physics by means of the two-channel case. We will provide numerical results and discuss their physical meaning in the following sections of the present paper.

\section{Numerical results}

\subsection{Eigenvalues and eigenfunctions of $\mathcal{H}^{(2,2)}$}

For illustration, we show in Figure 1 a few typical numerical results for two states in the two-channel case. The results of the left column are obtained for the special case $\omega^{(1)}=\omega^{(2)}$ and those of the right column for the general 


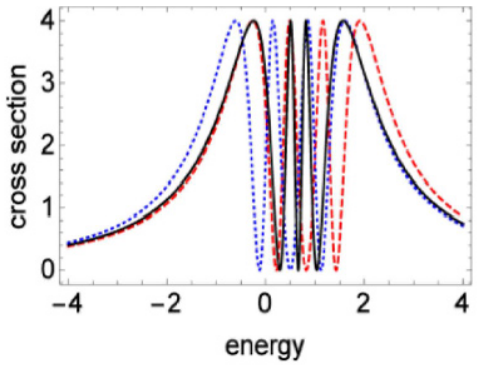

a

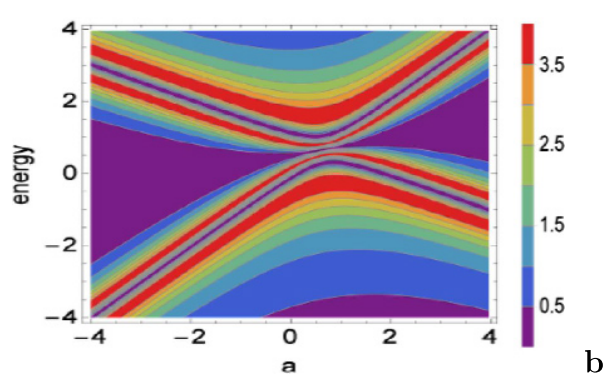

Fig. 2. (a) Resonance structure and (b) contour plot of the transmission with vanishing external mixing $\omega^{(1)}=\omega^{(2)}=0$. The parameters are the same as in Figure 1 (left). (a) full black line: $a=a^{\text {cr }}=0.6949$; dashed red line: $a_{1}=1.3$; dotted blue line: $a_{2}=0.0$

case $\omega^{(1)} \neq \omega^{(2)}$. The numerical results for the eigenvalue trajectories $\mathcal{E}_{i}^{(1,2)} \equiv E_{i}^{(1,2)}+\frac{i}{2} \Gamma_{i}^{(1,2)}$ and the eigenfunction trajectories $\Phi_{i}^{(1,2)}$ of the Hamiltonian $\mathcal{H}^{(2,2)}$ are obtained by starting from parameter-dependent energies $e_{i}^{(1,2)}$ and parameter-independent widths $\gamma_{i}^{(1,2)}$. We consider two different cases: in one case, the widths $\gamma_{i}^{(1,2)}$ of the two states are equal, $\gamma_{i}^{(1)}=\gamma_{i}^{(2)}\left(\right.$ correspondingly to $\left.\omega^{(1)}=\omega^{(2)}\right)$, while they are different from one another in the other case, $\gamma_{i}^{(1)} \neq \gamma_{i}^{(2)}$.

The eigenvalue trajectories Figures 1a, $1 \mathrm{~b}$ and 1f, 1g, respectively, show that the widths $\Gamma_{i}^{(1,2)}$ bifurcate in the neighborhood of an EP. The energies of the two states are equal in this parameter range. At the critical parameter value $a=a^{\mathrm{cr}}$ width bifurcation is maximum. Here the phase rigidity approaches the value 1 (Figs. 1d and 1i), meaning that the two states become orthogonal at this parameter value. The EM of the states via the continuum can not be neglected at this parameter value (Figs. 1e and $1 \mathrm{j}$ ).

While the eigenvalue trajectories of $\mathcal{H}^{(2,2)}$ are influenced by the critical point $a=a^{\text {cr }}$ mainly in its very neighborhood, the eigenfunction trajectories are influenced in a comparably large parameter range. This can be seen, above all, in the phase rigidity (Figs. 1c, 1d, 1h and 1i) which is a quantitative measure for the biorthogonality of the eigenfunctions of $\mathcal{H}^{(2,2)}$. This fact is known also from calculations for the one-channel case [11].

We underline here once more that the results shown in Figure 1 are obtained for $N=2$ resonance states. They show therefore boundary effects arising from the parameter range in which no resonance states exist. In realistic
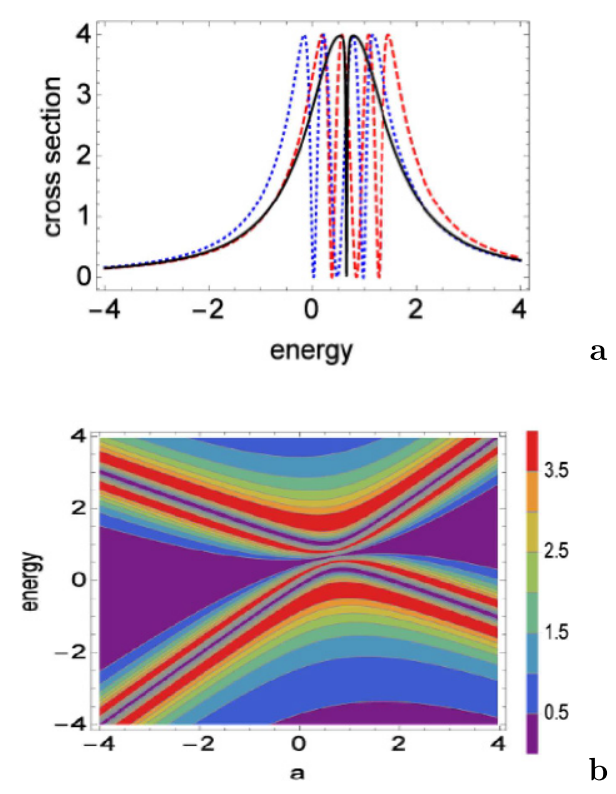

Fig. 3. The same as Figure 2 but $\omega^{(1)} \gg \omega^{(2)} \neq 0$. The parameters are the same as in Figure 1 (right).

systems, these boundary effects do, of course, not exist. Instead, we see an interference picture to which all $N$ states contribute, see e.g. [30,31].

\subsection{Resonance structure and contour plot of transmission}

Interesting information on the spectroscopic properties of the localized part of the system is contained in the resonance structure of the cross section which can be observed experimentally. We consider the transition from channel 1 to channel 2 which simulates transmission. Using (16), we have calculated the resonance structure of the transmission at the critical parameter value $a^{\mathrm{cr}}$ and at two values of $a$ beyond the critical range (above) as well as the corresponding contour plots (below).

In Figure 2, the results without EM (corresponding to $\omega^{(1)}=\omega^{(2)}=0$ ) are shown. There appear four states, indeed, according to the results of analytical studies. This picture is different from the resonance structure of the cross section of a two-level system coupled to one channel characteristic of which is its double-hump structure around maximum width bifurcation $[7,11]$.

Figures 3 and 4 show the transmission through a twolevel system coupled to two channels with $\omega^{(c)} \neq 0$ in the same parameter range around maximum width bifurcation. These figures illustrate that the double-hump structure of the cross section is restored in the transmission (i.e. in the two-channel case) under the influence of EM.

In Figure 3 (with different coupling strengths $\omega^{(1)} \gg$ $\omega^{(2)}$ according to the eigenvalues and eigenfunctions of Fig. 1 right), the double-hump structure around the maximum width distribution is very similar, indeed, to that of the cross section in the one-channel case (solid black line in Fig. 2 compared to solid black line in Fig. 4a in [11]). The 

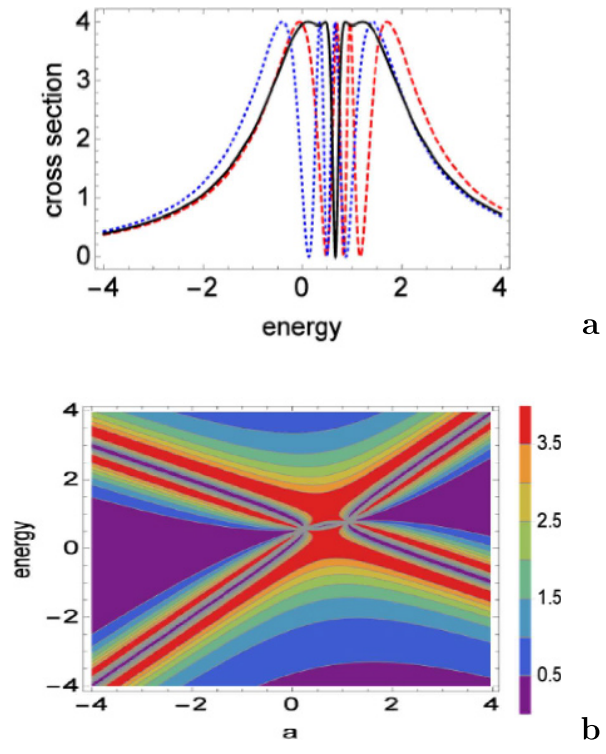

Fig. 4. The same as Figure 2 but $\omega^{(1)}=\omega^{(2)} \neq 0$. The parameters are the same as in Figure 1 (left).

contour plot of the transmission is however much richer than the cross section in the one-channel case. This is reflected also in the dotted (blue) and dashed (red) lines of Figure 3a.

More interesting are the results shown in Figure 4 corresponding to the eigenvalues and eigenfunctions of Figure 1 (left) with $\omega^{(1)}=\omega^{(2)}$. Here, the double-hump structure also appears. The bumps are however broadened in energy. Due to this broadening, the transmission is enhanced in a comparably large energy window. In this energy window, the phase rigidity averaged over energy, is reduced (see Fig. 1).

\section{Discussion of the results}

\subsection{External mixing}

The results shown in Figures 1-4 show very clearly that the mixing of the states via the environment (EM) plays an important role in open quantum systems. This mixing is a second-order effect and does not appear in standard Hermitian quantum physics. While its influence onto the spectroscopic properties of the localized part of the system far from singular points is usually small, it becomes important and determines the dynamics of the system in a finite neighborhood of the critical parameter values $a=a^{\mathrm{cr}}$.

\subsection{Meaning of "points"}

Our results show further that the dynamical properties of the system are influenced by EPs not only at parameter values that correspond to their "exact" position. Almost the same properties arise near to these parameter values. This can be seen, e.g., in the width trajectories in Figures $1 \mathrm{~b}$ and $1 \mathrm{~g}$ which bifurcate although the parameter values do not allow an exact crossing point of the trajectories.

\subsection{Two-channel systems and EPs}

According to the mathematical studies by Kato [20], EPs are defined in relation to one continuum. Their physical meaning is studied therefore, up to now, mostly in systems that are embedded in one well-defined common environment. For references see the review [7].

Most states of physical systems are coupled, however, to more than one channel. For example, the transmission through a small system (e.g. a quantum dot) is related to, at least, two channels, the entrance and the exit channel. The transmission of particles through such systems is studied by many authors in many different papers by using different methods. The most popular methods start from a Hermitian Hamilton operator and consider parameter ranges beyond EPs.

\subsection{Transmission}

In the present paper, transmission is studied for the first time in the framework of the non-Hermitian quantum physics without additional assumptions. The only assumption is that the (localized) system is embedded in more than one environment (meaning that it is coupled to more than one channel). Although the expression (13) for the Hamiltonian $\mathcal{H}^{(2,2)}$ seems to be arbitrary or a mathematical subtleness, the obtained results show that this genuine non-Hermitian Hamiltonian describes the properties of the two-channel system according to expectations.

\subsection{One-channel versus two-channel systems}

We compare the results obtained in the present paper for the two-channel system, with those known for open quantum systems embedded in one common environment. The following properties are independent of the number of channels.

(i) Far from singular points, the spectroscopic properties of the system are influenced only marginal by its embedding into an environment. They are, however, never exactly the same as those of a closed system. This fact is proven experimentally [27].

(ii) Singular points change the system properties in a certain finite neighborhood of their exact position. Here, they cause the same observable effects which are expected at their "exact" position.

(iii) At the critical parameter value $a^{\mathrm{cr}}$, the eigenfunctions of the non-Hermitian Hamilton operator $\mathcal{H}$ are orthogonal (and not biorthogonal). At this parameter value, width bifurcation is maximum; and, in the two-channel case, the transmission through the system is enhanced. This effect is observable.

The one-channel case has however some special features. The nonlinear processes involved in the non-Hermitian dynamics, restore the characteristic resonance structure of the cross section that has been obtained without taking into account EPs and EM. It is possible therefore to describe the system properties, in the one-channel case, without taking into account the characteristic features of 
an open quantum system [11]. The Figures 1-4 of our paper show clearly that this possibility is really restricted to the one-channel case. The information involved in the more-channel cases is much richer.

\subsection{Coupling strength to the two different channels}

In order to consider a system that is coupled really to two channels, the two coupling strengths $\omega^{(1)}$ and $\omega^{(2)}$ should be of comparable value. Otherwise the system is nothing but a one-channel system with a somewhat defective coupling strength. When $\omega^{(1)} \approx \omega^{(2)}$, the EPs in the two different channels appear at almost the same value of the parameter considered. In such a case it is possible to trace the influence of the EP onto observable values such as the cross section, compare Figure 3 with Figure 4 . The influence can be seen above all when $\omega^{(1)}=\omega^{(2)}$.

\subsection{Coherence in two-channel systems}

In the two-channel case EPs cannot be seen directly. We see only the critical value $a^{\text {cr }}$ of the parameter $a$ which gives rise to new observable effects such as the enhancement of the transmission at maximum width bifurcation. This effect is measurable. The phase rigidity is 1 at $a^{\text {cr }}$ because the eigenfunctions of $\mathcal{H}$ are orthogonal at this point. It is however $r_{k}<1$ in the neighborhood of $a^{\mathrm{cr}}$. Averaged over a certain parameter range around $a^{\mathrm{cr}}$, the phase rigidity is therefore reduced, while the transmission is enhanced.

In [13], the transmission through a system with many states is calculated numerically by using the tight-binding lattice model (according to Datta [33]) with one channel in each of the two attached identical leads. As a result of this calculation, the phase rigidity, averaged over the considered energy window, and the transmission are anticorrelated. Thus, the two very different methods (each of which solves the problem exactly) produce qualitatively the same results.

These results show that coherence appears in twochannel (and more-channel) systems. It appears additionally to dissipation which exists in every open quantum system. The EM is related to the environment and influences the different states of the system in the same manner, i.e. coherently. Finally, it causes the cross section broadening observed in Figure $4 .^{2}$

Coherence is known to play an important role in biophysical systems. According to our results, it is characteristic of all open quantum systems which are coupled to more than one open channel. It may be small; it is however nonvanishing.

We underline once more that the above mentioned processes are related exclusively to the properties of the eigenstates of a non-Hermitian Hamilton operator. They are not involved in any version of Hermitian quantum physics.

\footnotetext{
${ }^{2}$ We underline here once more that the cross section broadening observed in the present paper is not caused by an enhancement of the coupling strength $\omega$ between system and environment (in difference to the results of some earlier calculations). The value of $\omega$ is kept constant in each calculation, as can be seen from the captions of the figures.
}

For example, EM can be simulated, to some extent, in the standard calculations with Hermitian Hamiltonian by including it effectively into the Hamiltonian. Its interesting relation to the critical parameter value $a^{\text {cr }}$ can, however, not be seen in such calculations.

\section{Concluding remarks}

Our results show that the genuine non-Hermitian Hamiltonian $\mathcal{H}$ can be used for the description of the characteristic features of many-body systems with transfer of particles. In this case, the Hamiltonian $\mathcal{H}$ is surely not the most convenient one in order to describe a realistic system. It demonstrates however that non-Hermitian quantum physics is a powerful method that can explain many different features of open quantum systems, including those that can be described successfully in standard theory.

It is very well known that the non-Hermitian part of the Hamiltonian causes dissipation, which is characteristic of all open quantum systems. In competition with dissipation, coherence appears when the system is coupled to at least two open channels. The competition between coherence and dissipation becomes the more important the more channels are open. It is the most interesting feature of realistic open quantum systems.

The results of the present paper show furthermore that the eigenfunctions of the non-Hermitian Hamilton operator $\mathcal{H}$ play an important role, above all their non-rigid phases around the singular EPs; and their non-vanishing external mixing via the environment. At the critical parameter value $a^{\mathrm{cr}}$, a short-lived state results from width bifurcation. This state is similar to the so-called superradiant state discussed in, e.g., [16,17]. The mechanism of the formation of the superradiant state is however completely different from that of the short-lived state discussed in the present paper.

The critical point $a^{\mathrm{cr}}$ at which the eigenfunctions of the non-Hermitian Hamilton operator $\mathcal{H}$ are orthogonal (and not biorthogonal), appears in the one-channel as well as in the two-channel problem (for the one-channel case see [11]). Here, the quantum system and the environment into which it is embedded, are in a state of equilibrium [34].

The same formalism of non-Hermitian quantum physics that is able to describe properties of many-particle systems, can explain also experimental results which are puzzling in standard Hermitian quantum physics. Examples are the explanation [35] of the so-called phase lapses in mesoscopic systems as well as the understanding [28] for the high efficiency of photosynthesis.

Summarizing we state that open quantum systems embedded in two (or more) environments are abounded in very many interesting aspects of non-Hermitian quantum physics. They allow, on the one hand, to study characteristic features of non-Hermitian quantum physics. On the other hand, they provide the possibility of many promising applications.

We are indebted to Jon Bird for valuable discussions. Open access funding provided by Max Planck Society. 


\section{Author contribution statement}

H.E. has performed the numerical calculations while the text of the paper is written mainly by I.R.

Open Access This is an open access article distributed under the terms of the Creative Commons Attribution License (http://creativecommons.org/licenses/by/4.0), which permits unrestricted use, distribution, and reproduction in any medium, provided the original work is properly cited.

\section{References}

1. H. Friedrich, D. Wintgen, Phys. Rev. A 31, 3964 (1985)

2. H. Friedrich, D. Wintgen, Phys. Rev. A 32, 3231 (1985)

3. I. Rotter, A.F. Sadreev, Phys. Rev. E 71, 046204 (2005)

4. E.N. Bulgakov, A.F. Sadreev, Phys. Rev. A 94, 033856 (2016), and references therein

5. Y. Yoon, M.-G. Kang, T. Morimoto, M. Kida, N. Aoki, J.L. Reno, Y. Ochiai, L. Mourokh, J. Fransson, J.P. Bird, Phys. Rev. X 2, 021003 (2012)

6. S. Xiao, Y. Yoon, Y.-H. Lee, J.P. Bird, Y. Ochiai, N. Aoki, J.L. Reno, J. Fransson, Phys. Rev. B 93, 165435 (2016)

7. I. Rotter, J. Phys. A 42, 153001 (2009)

8. H. Eleuch, I. Rotter, Eur. Phys. J. D 69, 229 (2015)

9. H. Eleuch, I. Rotter, Phys. Rev. A 93, 042116 (2016)

10. H. Eleuch, I. Rotter, Eur. Phys. J. D 69, 230 (2015)

11. H. Eleuch, I. Rotter, Phys. Rev. A 95, 022117 (2017)

12. E.N. Bulgakov, I. Rotter, A.F. Sadreev, Phys. Rev. E 74, 056204 (2006)

13. E.N. Bulgakov, I. Rotter, A.F. Sadreev, Phys. Rev. B 76, 214302 (2007)
14. R.G. Nazmitdinov, K.N. Pichugin, I. Rotter, P. Seba, Phys. Rev. E 64, 056214 (2001)

15. R.G. Nazmitdinov, K.N. Pichugin, I. Rotter, P. Seba, Phys. Rev. B 66, 085322 (2002)

16. G.L. Celardo, A. Biella, L. Kaplan, F. Borgonovi, Fortschr. Phys. 61, 250 (2013)

17. G.L. Celardo, L. Kaplan, Phys. Rev. B 79, 155108 (2009)

18. T.J.G. Apollaro, S. Lorenzo, C. Di Franco, F. Plastina, M. Paternostro, Phys. Rev. A 90, 012310 (2014)

19. A. Sergi, P.V. Giaquinta, Entropy 18, 451 (2016)

20. T. Kato, Perturbation Theory for Linear Operators, (Springer Berlin, 1966)

21. I. Rotter, Phys. Rev. E 64, 036213 (2001)

22. A.I. Magunov, I. Rotter, S.I. Strakhova, J. Phys. B 34, 29 (2001)

23. U. Günther, I. Rotter, B.F. Samsonov, J. Phys. A 40, 8815 (2007)

24. B. Wahlstrand, I.I. Yakimenko, K.F. Berggren, Phys. Rev. E 89, 062910 (2014)

25. F. Tellander, K.F. Berggren, Phys. Rev. A 95, 042115 (2017)

26. Y.V. Fyodorov, D.V. Savin, Phys. Rev. Lett. 108, 184101 (2012)

27. J.B. Gros, U. Kuhl, O. Legrand, F. Mortessagne, E. Richalot, D.V. Savin, Phys. Rev. Lett. 113, 224101 (2014)

28. H. Eleuch, I. Rotter, Phys. Rev. E 95, 062109 (2017)

29. I. Rotter, J.P. Bird, Rep. Prog. Phys. 78, 114001 (2015)

30. P. Kleinwächter, I. Rotter, Phys. Rev. C 32, 1742 (1985)

31. I. Rotter, Rep. Prog. Phys. 54, 635 (1991)

32. I. Rotter, Phys. Rev. E 68, 016211 (2003)

33. S. Datta, Electronic Transport in Mesoscopic Systems, (Cambridge University Press, Cambridge, 1995)

34. I. Rotter, Entropy 20, 441 (2018)

35. M. Müller, I. Rotter, Phys. Rev. A 80, 042705 (2009) 